\title{
FUNCTIONAL TRIMMING OF THICK-FILM ACTIVE FILTERS FOR CONSUMER AND INDUSTRIAL APPLICATIONS
}

\author{
GIUSEPPE DELL'ORTO and GUGLIELMO RAVASCHIO \\ Magneti Marelli - Systems and Electronics Division-Pavia (Italy)
}

(Received March 13, 1980)

\begin{abstract}
Active filters, realized in thick film circuitry, can offer major advantages over more conventional wiring systems, including the ability to functionally trim them, using a computerized laser trimming system. This paper considers the adjustment methods used for two different active filters and shows how the methods can be extended to filters with more complicated characteristics.
\end{abstract}

\section{INTRODUCTION}

Active filters made on printed circuit boards require high-precision components or critical manual adjustments of resistor trimmers to tune each single stage, with many problems in reproducibility and reliability.

Such disadvantages can be successfully removed by thick film technology, using automatic functional trimming by a computerized laser system.

The implementation of a suitable interface box allows a laser system, originally designed for dc trimming, to adjust RC time constants, gain and phase variations of thick film active filters. In this way, filter trimming has been turned into a fast and repetitive process, with very good results in high volume production. This paper deals with the adjustment methods used in the production of two active filters for quite different applications, as electronic musical instruments and automatic controls for industrial use.
The former, required above all, manufacturing costs to be reduced as much as possible, the latter required rather critical specifications to be met with great accuracy.

It will be pointed out how the interface system designed for the consumer filter (already described in another paper) can be improved with simple criteria, to adjust also filters with more sophisticated characteristics.

\section{FILTER FOR MUSICAL INSTRUMENTS APPLICATION}

\subsection{Schematic Diagram}

The manufacturers of electronic musical instruments use a large number of "octave filters" to get pure sinusoidal signals from square wave generators. Because of very tight cost constraints, filters are usually made on printed

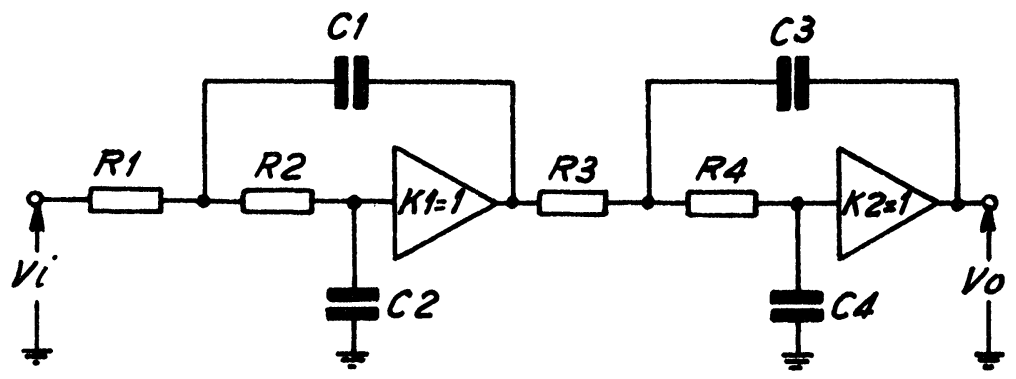

FIGURE 1 Sallen-Key filter structure. 


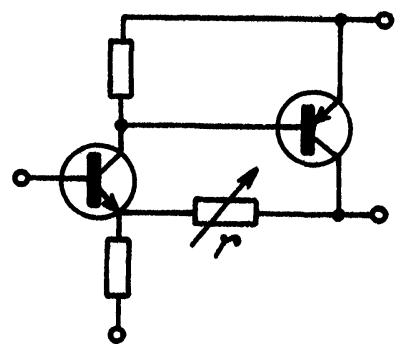

FIGURE 2 Active element in Sallen-Key filter.

circuit boards but high-precision capacitors and a manual individual adjustment are not always sufficient to generate sounds of acceptable and constant quality from assembled instruments.

Thick film active filters replace older versions with advantages in cost and performance, thanks to a proper circuits structure, which includes general-purpose transistors and large-tolerance capacitors and is suitable for functional automatic trimming.

The filter circuit is based on the well-known Sallen-Key structure (Figure 1). The active element in each stage is a unity-gain amplifier, implemented with two transistors (Figure 2). The transfer function is

$\frac{\bar{V}_{0}}{\bar{V}_{i}}=\frac{K_{1}}{R_{1} R_{2} C_{1} C_{2} p^{2}+\left[R_{1} C_{2}+R_{2} C_{2}+R_{1} C_{1}\left(1-K_{1}\right)\right] p+1}$.

$\frac{K_{2}}{R_{3} R_{4} C_{3} C_{4} p^{2}+\left[R_{3} C_{4}+R_{4} C_{4}+R_{3} C_{3}\left(1-K_{2}\right)\right] p+1}$

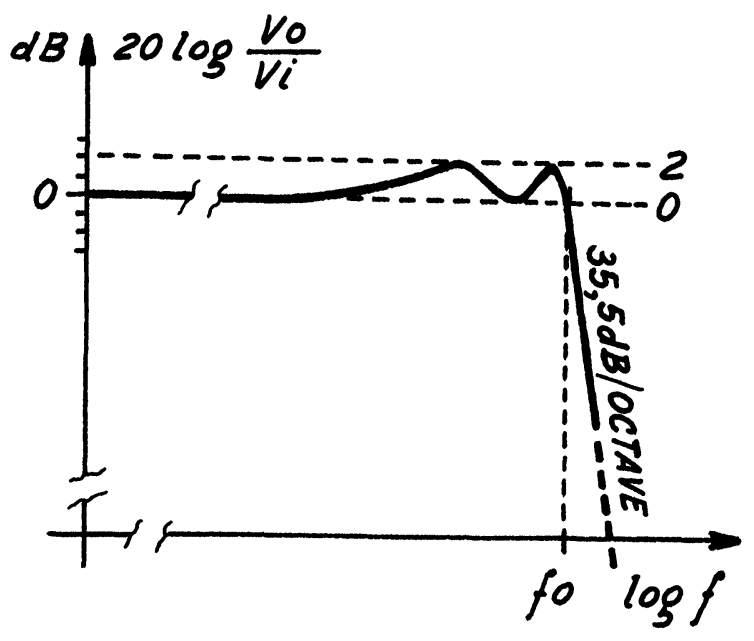

FIGURE 3 Frequency response of filter. where polynomial coefficients have been dimensioned to give a Chebysheff low-pass characteristic of fourth order.

The frequency response is outlined in Figure 3, showing a ripple of $2 \mathrm{~dB}$ in the pass band and an attenuation of about $35,5 \mathrm{~dB} /$ octave in the stop band, considered quite satisfactory for musical instruments manufacturers.

\subsection{Functional Trimming}

The actual reproduction of the calculated filter characteristic requires the trimming of frequency response in each stage, that is the polynomial coefficients of Eq. 1 must reach their theoretical values. With the first step, time constants $R_{1} C_{1}$, $R_{2} C_{2}$ of the first stage and $R_{3} C_{3}, R_{4} C_{4}$ of the second stage are functionally adjusted, to reach the nominal values of $p^{2}$ coefficients. In the second step, gains $K_{1}$ and $K_{2}$ must be adjusted also to compensate for errors deriving from cross terms $R_{1} C_{2}$ and $R_{3} C_{4}$ and this is achieved by trimming resistors $r$ (Figure 2), affecting the stage gains. Since $R_{1} C_{1} \gg R_{1} C_{2}$ and $R_{3} C_{3} \gg R_{3} C_{4}$, very small deviations of $K_{1}$ and $K_{2}$ close to the unity value are sufficient to obtain the desired adjustment.

The block diagrams of the interface device are shown in Figures 4 and 5.

In the first section, a voltage generator $G$ supplies a sinusoidal signal $V_{G}$ to the network $R_{X} C_{X}$ under trimming and to a reference network $R_{R} C_{R}$, with nominal value time constant.

Their outputs are connected, through two peak-detectors, to the trimming system, which compares the two d.c. voltage levels and stops cutting resistor $R_{X}$ when $\left|V_{A}\right|=\left|V_{B}\right|$, that is

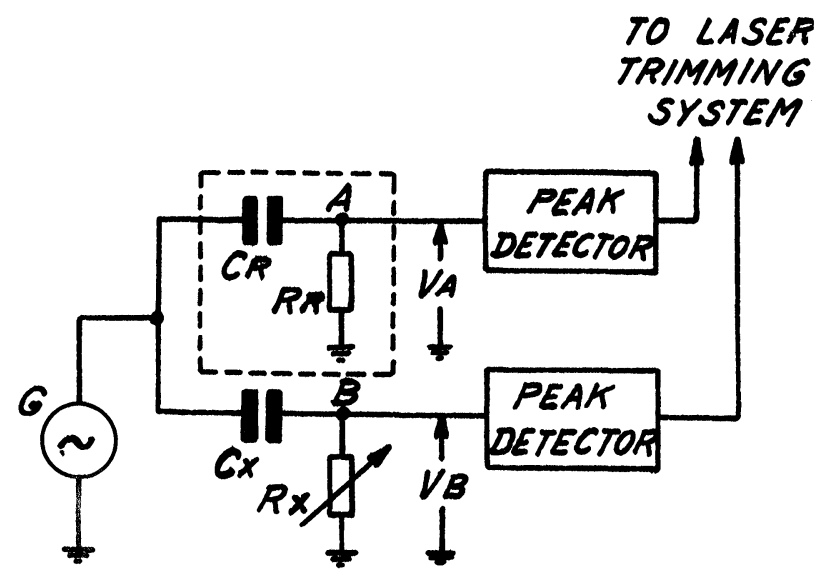

FIGURE 4 Block diagram of interface to laser (No. 1). 


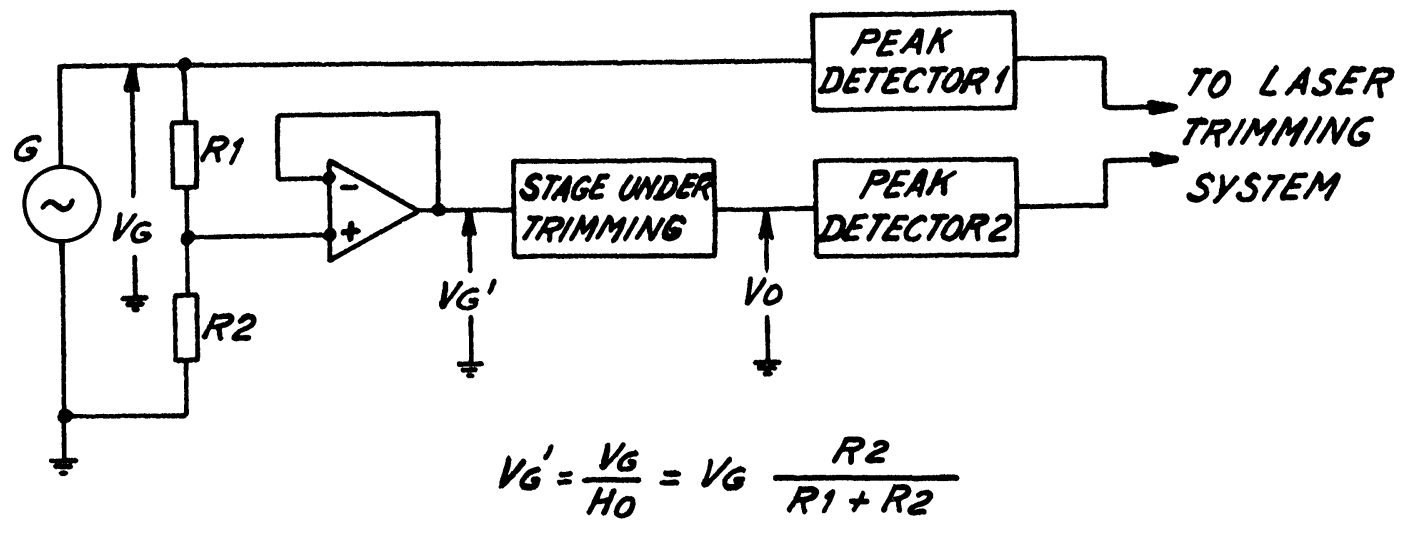

FIGURE 5 Block diagram of interface to laser (No. 2).

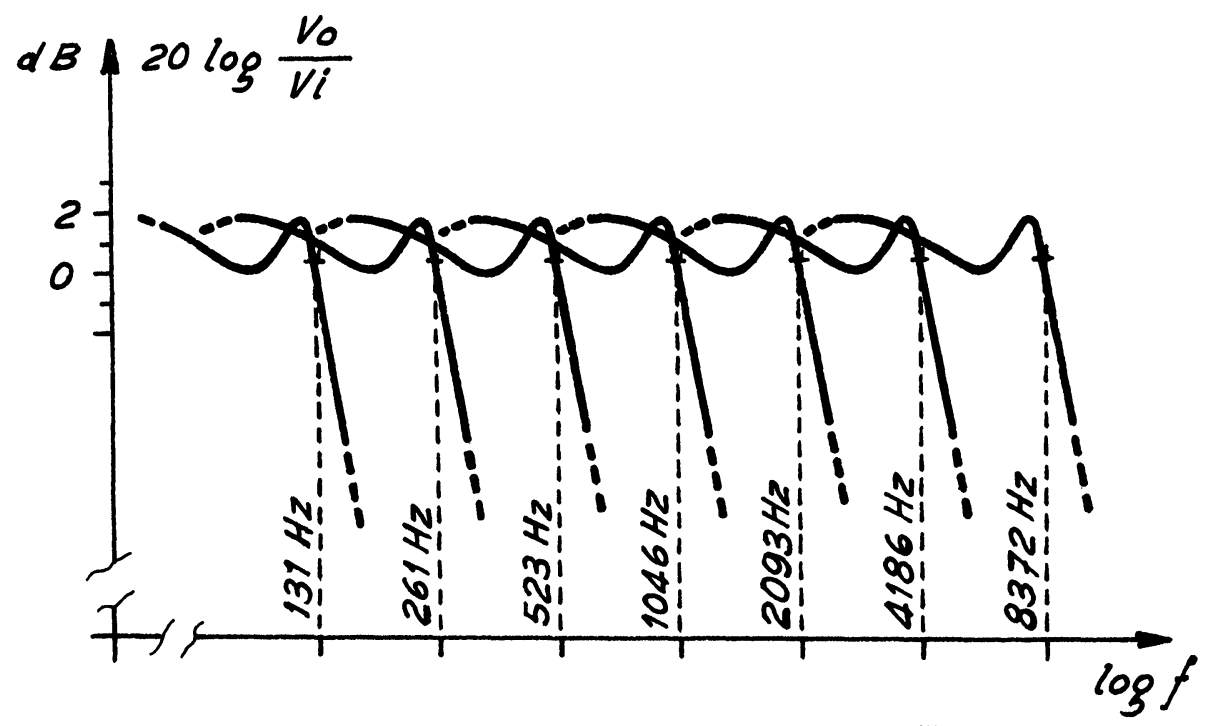

FIGURE 6 Frequency responses of electronic organ filters.

$R_{X} C_{X}=R_{R} C_{R}$. In the second section, a sinusoidal signal at the stage tuning frequency, is sent to peak-detector 1 directly and to peak-detector 2 through a divider, with ratio equal to $H$ and through the stage to be adjusted. The d.c. voltage levels from the peak detectors are supplied to the trimming system which cuts resistor $r$ until the stage gain reaches the nominal value $H$.

The most important advantage of this comparison method lies in its precision and reproducibility, not affected by precision and stability of the voltage generator $G$.
An electronic musical organ usually includes seven filters with seven different cut-off frequencies, ranging from $100 \mathrm{~Hz}$ to $8500 \mathrm{~Hz}$.

The complete kit is obtained from two modules, using the same set of masks, with changes of software only.

The basic thick-film module is screen-printed on a $38.1 \times 16.9 \mathrm{~mm}$. ceramic substrate. Add-on components are four general purpose transistors in SOT23 case and four multi-layer ceramic chip capacitors with a tolerance of $\pm 20 \%$.

Figure 6 describes the frequency responses of 


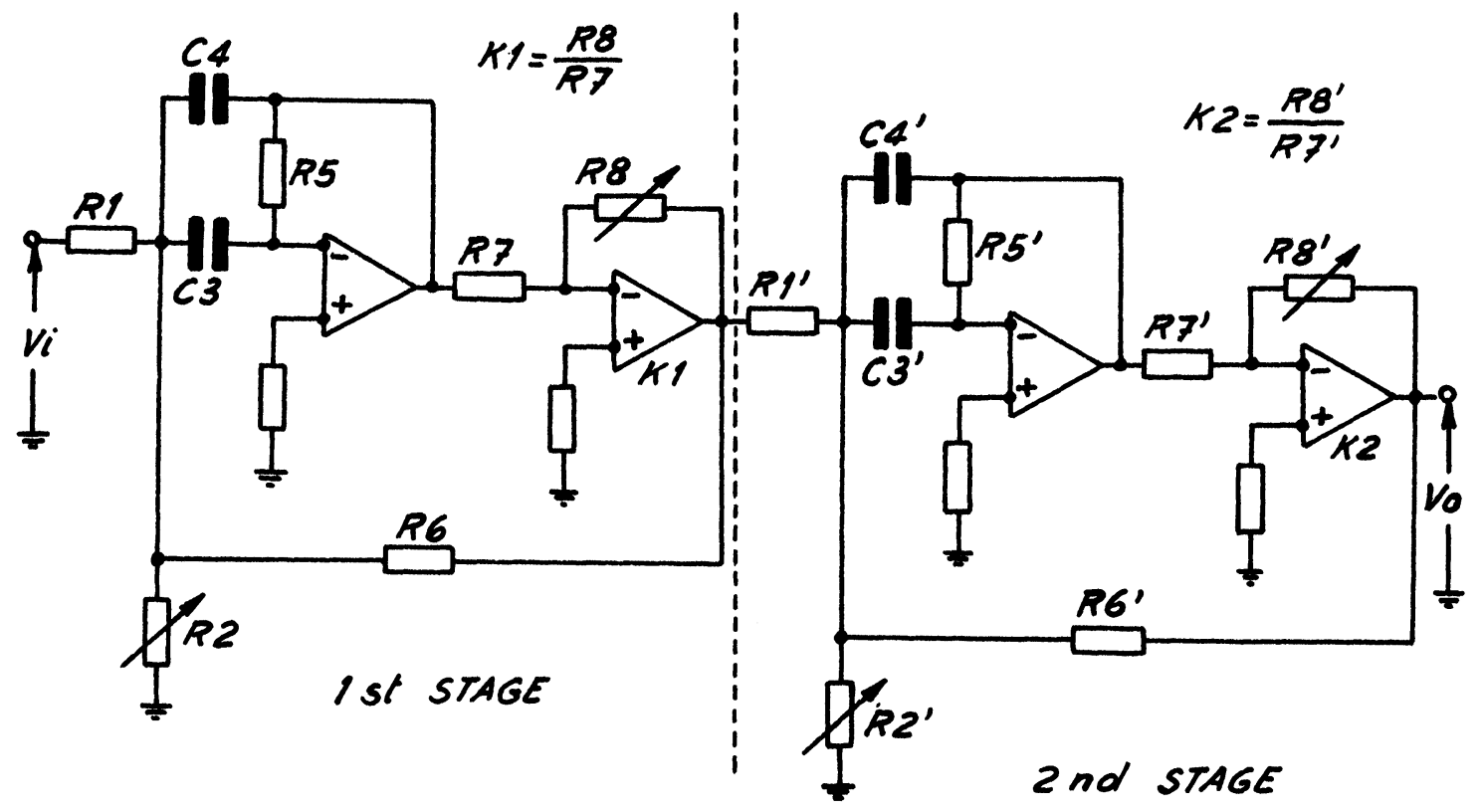

FIGURE 7 Fourth order pass-band filter.

the seven filters now in production. They differ in cut-off frequencies only, which can be also changed on customers specification.

The following table gives the comparison between traditional and thick-film filters:

TABLE I

Comparison between traditional and thick-film filters

\begin{tabular}{lll}
\hline Characteristic & P.C. Board & T.F. Module \\
\hline Precision in cut-off frequency & $\pm 2 \%$ & $\pm 0,5 \%$ \\
Max. ripple in the pass band & $3,5 \mathrm{~dB}$ & $2,4 \mathrm{~dB}$ \\
Min. attenuation in the stop band & $30 \mathrm{~dB}$ & $34,5 \mathrm{~dB}$ \\
\hline
\end{tabular}

\section{FILTER FOR INDUSTRIAL APPLICATION}

\subsection{Filter Circuit and Characteristics}

This filter is a fourth order pass-band type, including two stages with multiple feed-back (Figure 7). It has been designed with a monolithic quad op. amp. of middle quality (gain $x$ band width $\simeq 3 \mathrm{MHz}$ ) and NPO ceramic chip capacitors, with tolerance of $\pm 5 \%$. Its transfer function is:

$$
\frac{\bar{V}_{0}}{\bar{V}_{i}}=\frac{\frac{K_{1} R_{5} C_{3}}{R_{1}\left[\frac{1}{R_{1}}+\frac{1}{R_{2}}+\frac{1}{R_{6}}\right]} \cdot p}{\frac{C_{3} C_{4} C_{5}}{\frac{1}{R_{1}}+\frac{1}{R_{2}}+\frac{1}{R_{6}}} \cdot p^{2}+\frac{C_{3}\left[1+\frac{C_{4}}{C_{3}}-K_{1} \frac{R_{5}}{R_{6}}\right]}{\frac{1}{R_{1}}+\frac{1}{R_{2}}+\frac{1}{R_{6}}} \cdot p+1}
$$

$$
\frac{K_{2} R_{5} C_{3}^{\prime}}{R_{1}^{\prime}\left[\frac{1}{R_{1}^{\prime}}+\frac{1}{R_{2}^{\prime}}+\frac{1}{R_{6}^{\prime}}\right]} \cdot p
$$

$\frac{C_{3}^{\prime} C_{4}^{\prime} R_{5}^{\prime}}{\frac{1}{R_{1}^{\prime}}+\frac{1}{R_{2}^{\prime}}+\frac{1}{R_{6}^{\prime}}} \cdot p^{2}+\frac{C_{3}^{\prime}\left[1+\frac{C_{4}^{\prime}}{C_{3}^{\prime}}-K_{2} \frac{R_{5}^{\prime}}{R_{6}^{\prime}}\right]}{\frac{1}{R_{1}^{\prime}}+\frac{1}{R_{2}^{\prime}}+\frac{1}{R_{6}^{\prime}}} \cdot p+1$

The frequency response is outlined in Figure 8 and is expressed, in normalized form, by the equation:

$$
F\left(P_{0}\right)=\frac{c_{1} p_{0}}{a_{1} p_{0}^{2}+b_{1} p_{0}+1} \cdot \frac{c_{2} p_{0}}{a_{2} p_{0}^{2}+b_{2} p_{0}+1}
$$




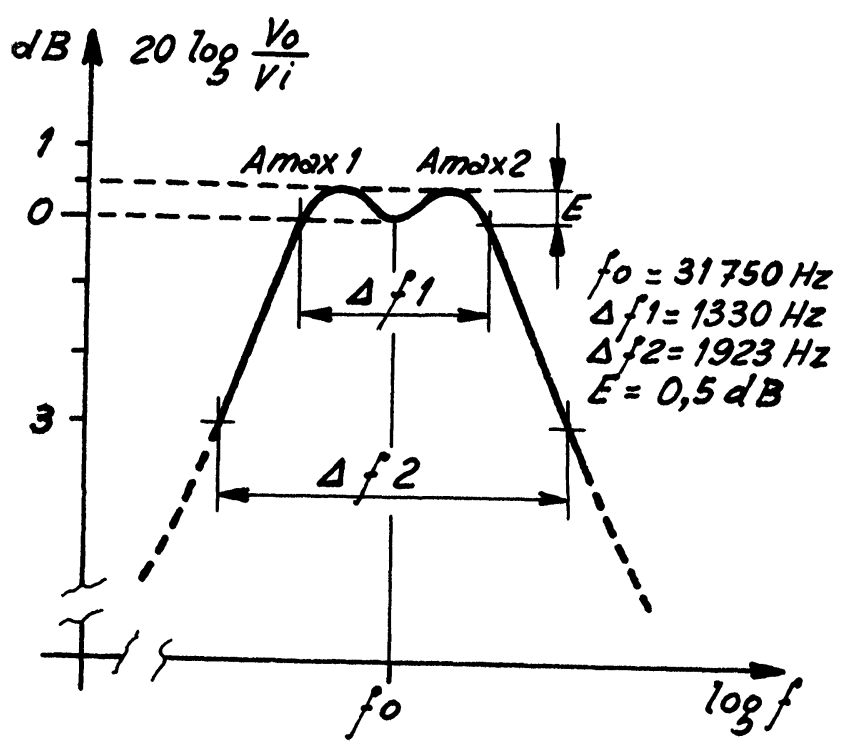

FIGURE 8 Frequency response of pass-band filter.

where:

$a_{1}=1,04296, b_{1}=3,04874 \cdot 10^{-2}, c_{1}=5,15806 \cdot 10^{-2}$. $a_{2}=9,58811 \cdot 10^{-1}, b_{2}=2,92317.10^{-2}$, $c_{2}=5,15806 \cdot 10^{-2}$.

The two filter stages are defined by their own frequency responses, plotted in Figures 9 and 10.

Tuning frequencies, practically coinciding with

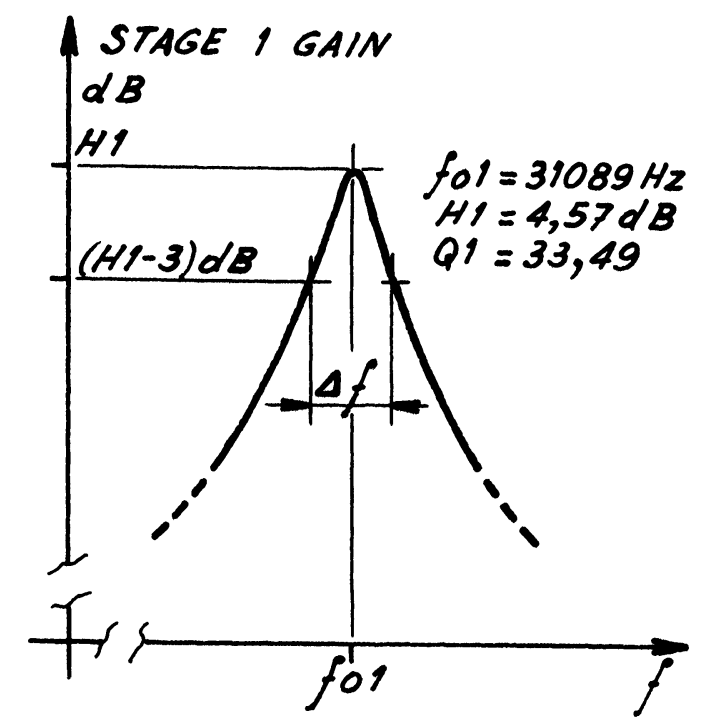

FIGURE 9 Frequency response of first filter stage. maximum points, may be written as:

$$
f_{0 i}=f_{0} / \sqrt{a_{i}}
$$

where

$$
i=1,2
$$

and then

$$
H_{i}=c_{i} / b_{i} \quad Q_{i}=\sqrt{a_{i}} / b_{i}
$$

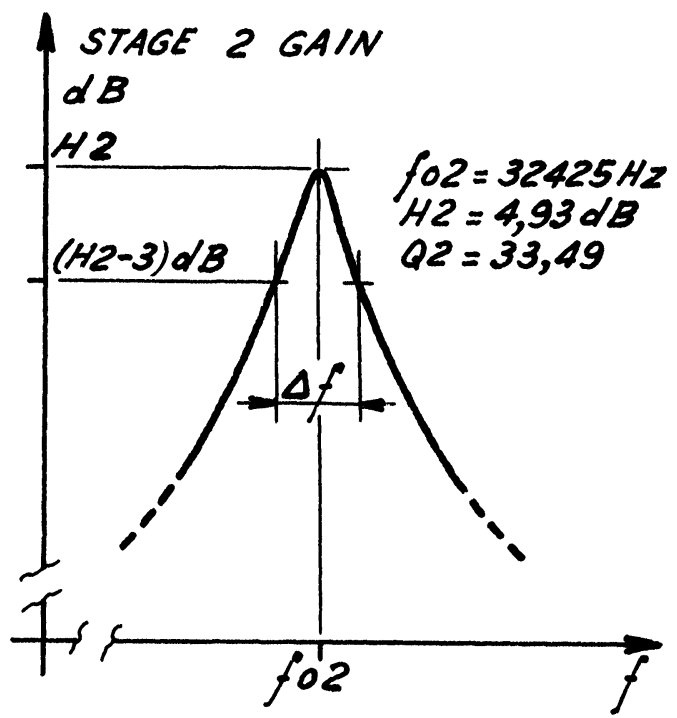

FIGURE 10 Frequency response of second filter stage.

\subsection{Functional Trimming: Principle of Operation}

In each stage, at its tuning frequency, there is no phase deviation between input and output signals. Eq. (2) and (4) show that frequencies and phasedeviations of the two stages depend on $a_{1}$ and $a_{2}$, $p_{2}$-coefficients in the transfer function. Thus we can tune each stage at its own frequency, by trimming resistors $R_{2}$ and $R_{2}^{\prime}$, stopping the laser trimmer when phase deviation becomes zero and $p^{2}$-coefficients reach the nominal values.

We can then proceed to adjust gains $H_{i}$, according to Eq. (5). Coefficients $b_{1}, c_{1}, b_{2}$ and $c_{2}$ could not be exactly equal to their respective theoretical values but computer analysis shows that deviations are negligible and cause a small vertical translation of the curve in Figure 8, without affecting filter selectivity. 


\subsection{Functional Trimming: Practical Implementation}

The interface system is designed to use the standard d-c type computer controlled laser trimmer.

The block diagram of the phase-control section is shown in Figure 11, where $V_{i}$ is a sinusoidal signal, supplied to this circuit and to the filter stage under trimming at the same time. Figure 12a shows $V_{i}$ and $V_{0}$ before trimming, which produce the waveforms $V_{1}, V_{2}$ and $V_{3}$ of Figures $12 \mathrm{~b}, \mathrm{c}$, d in the interface network. When the phases of $V_{0}$ and $V_{i}$ became equal, we have at the same instant $V_{2}=V_{3}=$ "1" and $V_{4}=$ " 0 ". At this moment, the output $V_{5}$ from the memory unit goes up to " 1 " and stops the laser, trimming resistor $R_{2}$ (or $R_{2}^{\prime}$ ).

Note that the one-shot no 2 is connected to similarly operate the AND-gate, to have the same time-delay for signals in the two paths towards the laser control input and, in this way, to improve the system precision.

This simple solution offers remarkable accuracy in operation, with response times in the order of a few nanoseconds. The interface section to control gain adjustments is the same previously described for the consumer filter. The right values of gains are
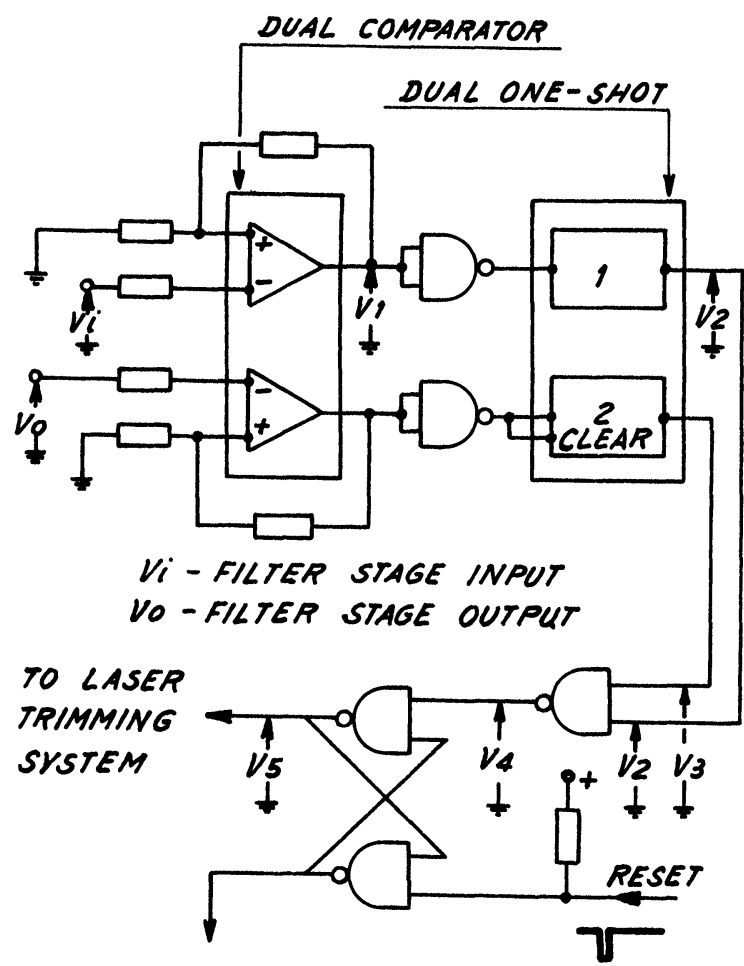

SEQUENTIAL CIRCUIT

FIGURE 11 Block diagram of phase control section.

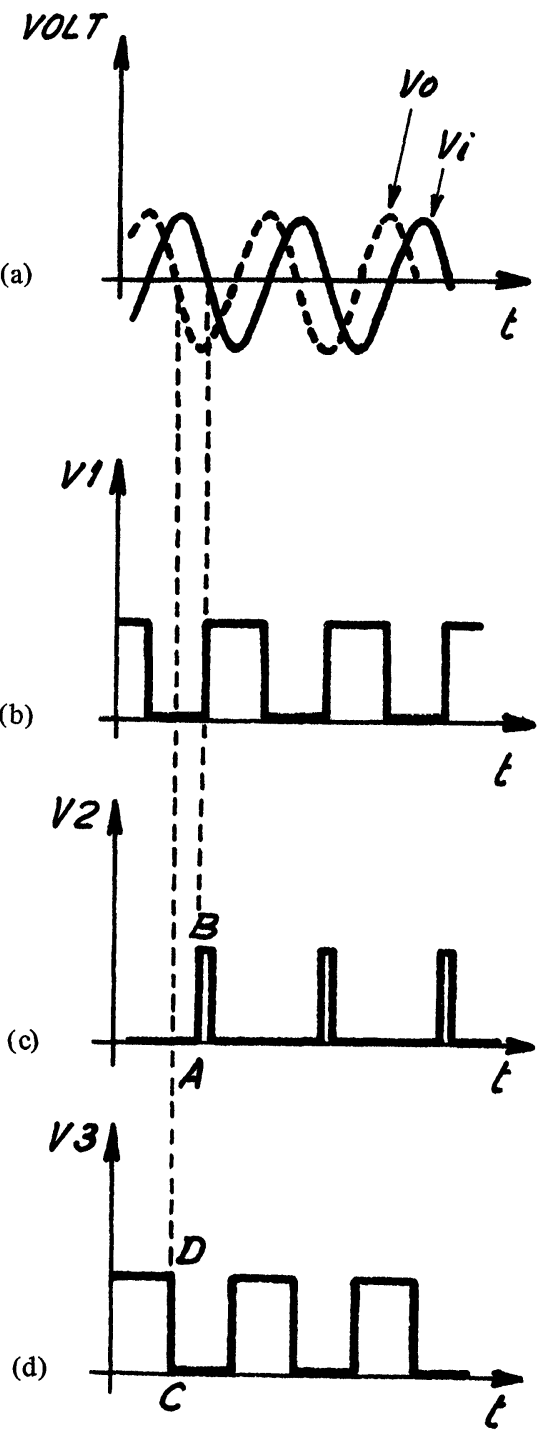

FIGURE 12 (a) - (d). Voltage waveforms in the phase control circuit.

reached by trimming resistors $R_{8}$ and $R_{8}^{\prime}$ (Figure 7), directly affecting $K_{1}$ and $K_{2}$ parameters.

Actually, phase and gain adjustments are not independent at all but affect each other in a small amount, because of effective limitations in op. amp. characteristics, particularly gain bandwidth product. To compensate for the consequent error, the adjustment of each stage is achieved through four steps, the first two of which are a coarse calibration and the second two are a fine calibration. This is described in Figure 13, concerning the first filter stage. First of all, the circuit is tuned at a frequency 


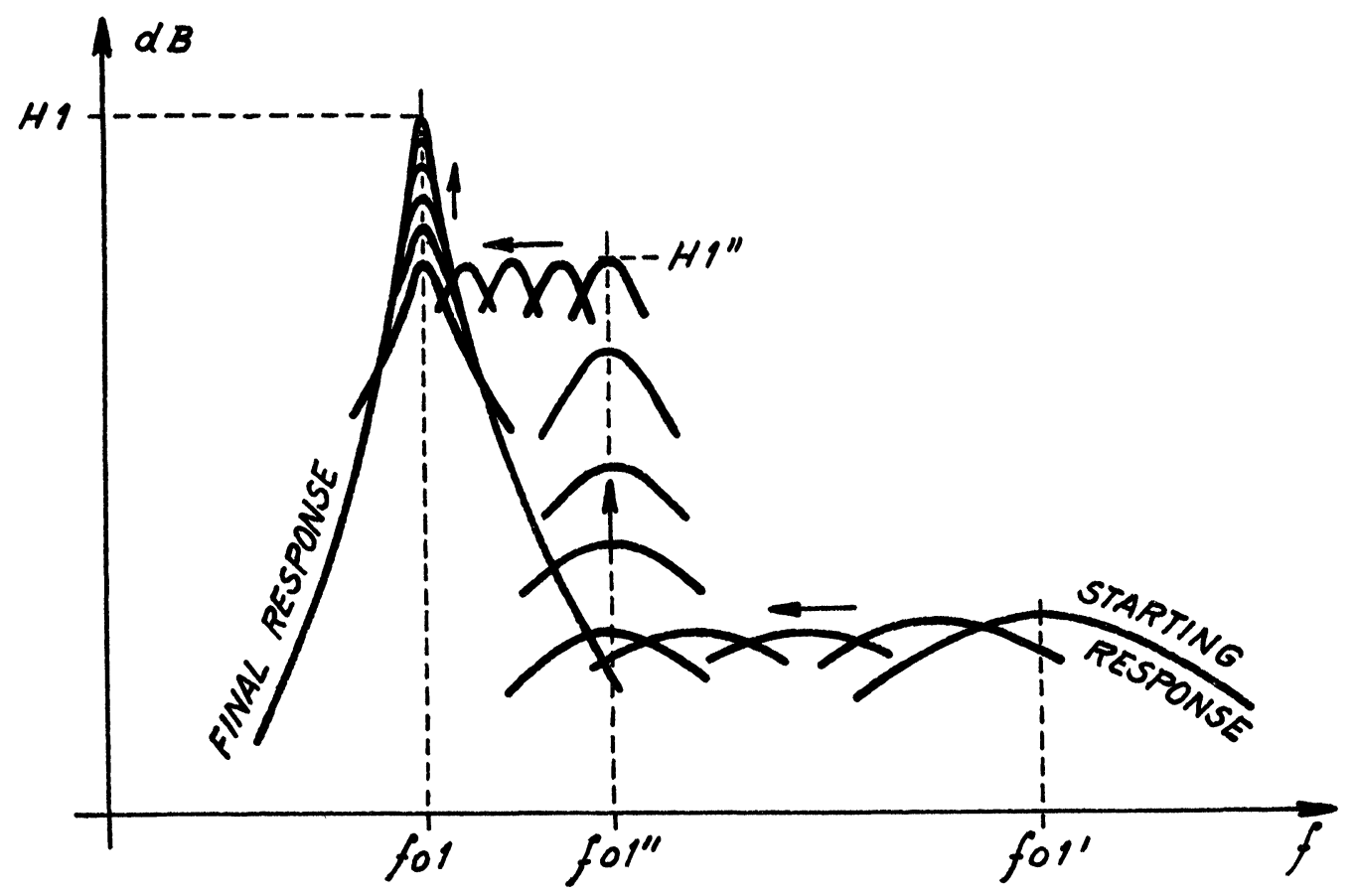

FIGURE 13 Diagram showing stages of the first filter adjustment.

$f_{0 i}{ }^{\prime \prime}<f_{0}$, then the gain is brought to a value $H_{1} "<H_{1}$ and finally, by two other similar steps, final values $f_{01}$ and $H_{01}$ are reached.

\subsection{Hybrid Module Characteristics}

The circuit is assembled on a ceramic substrate of $22,4 \times 38,1 \mathrm{~mm}$., screen-printed on both sides.

Functional trimming resistors have rather large dimensions, to get final values with several cuts without generating problems in noise and stability.

After functional trimming, modules are packaged into a plastic case filled with epoxy resin and then tested with an automatic computerized process.

The following table gives main electrical characteristics with production tolerances:

$$
\begin{aligned}
& f_{0}=31,750 \mathrm{kHz} \pm 45 \mathrm{~Hz} \\
& A_{0}=0 \mathrm{~dB} \pm 0,3 \mathrm{~dB} \\
& \Delta f_{1}=1330 \mathrm{~Hz} \pm 80 \mathrm{~Hz} \\
& \Delta f_{2}=1923 \mathrm{~Hz} \pm 70 \mathrm{~Hz} \\
& \left|\operatorname{Amax}_{1}-A_{m_{2}}\right| \leqslant 0,3 \mathrm{~dB}
\end{aligned}
$$

\section{CONCLUSION}

The thick film module of the pass-band filter described in this paper is a good compromise in performances, accuracy, dimensions and price. Other electrical circuits would be more suitable for adjustment and accuracy but would require one amplifier more in each stage.

The above-mentioned method of functional trimming combines a system of simple implementation with very good operation in the production process and, moreover, can be extended to other kinds of filters.

The opportunity of performing functional adjustment with several steps of increasing accuracy allows more critical circuits to be tuned.

\section{REFERENCES}

1. Dell'Orto, Ravaschio, "A low cost thick-film active filter for consumer applications," E.C.C. Proc. 1978, Anaheim (Ca-USA).

2. Sallen, Key, "A practical method of designing RC active filters," IRE Trans. CT 2 (1) 1955.

3. Bidstein, Filters actifs, Ed. Radio, Paris 1972.

4. Temes, Mitra, Modern filter theory and design, J. Wiley and Sons, New York 1973.

5. Huelsman, Active RC filters: Theory and application, Dowden, Hutchinson and Ross, Stroutsburg (Pa-USA) 1976.

6. Huelsman, Active filters, lumped, distributed, digital and parametric, McGraw-Hill, New York 1970.

7. Tobey, Graeme, Huelsman, Operational amplifiers, McGraw-Hill, New York 1971. 
8. Active filters, $A F 100$ universal active filters, National Semiconductor 1976

9. Wang, Ott, Function circuits, McGraw-Hill, New York 1976.
10. Graeme, Applications of op. amplifiers, McGraw-Hill, New York 1973.

11. Graeme, Designing with op. amplifiers, McGraw-Hill, New York 1977. 

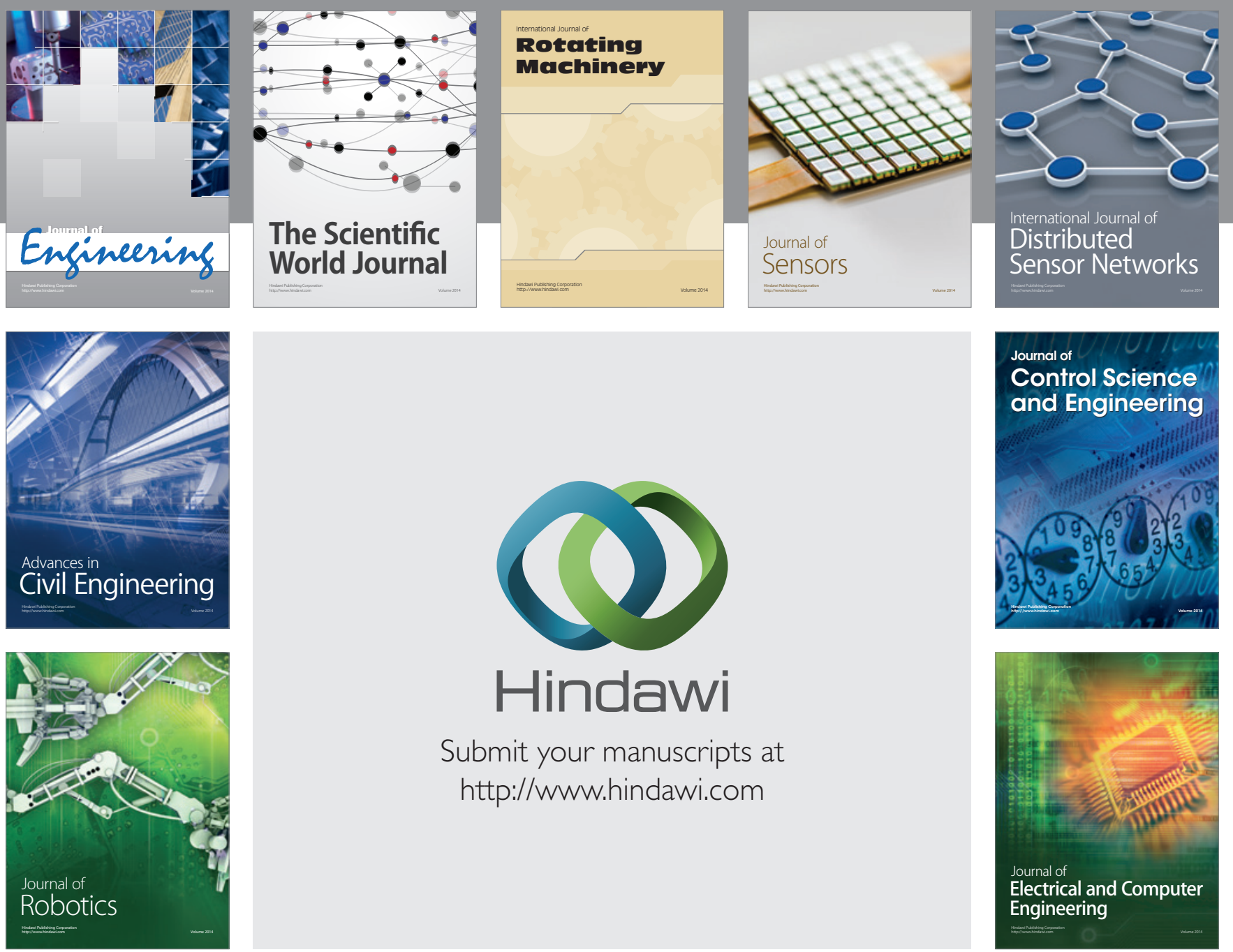

Submit your manuscripts at

http://www.hindawi.com
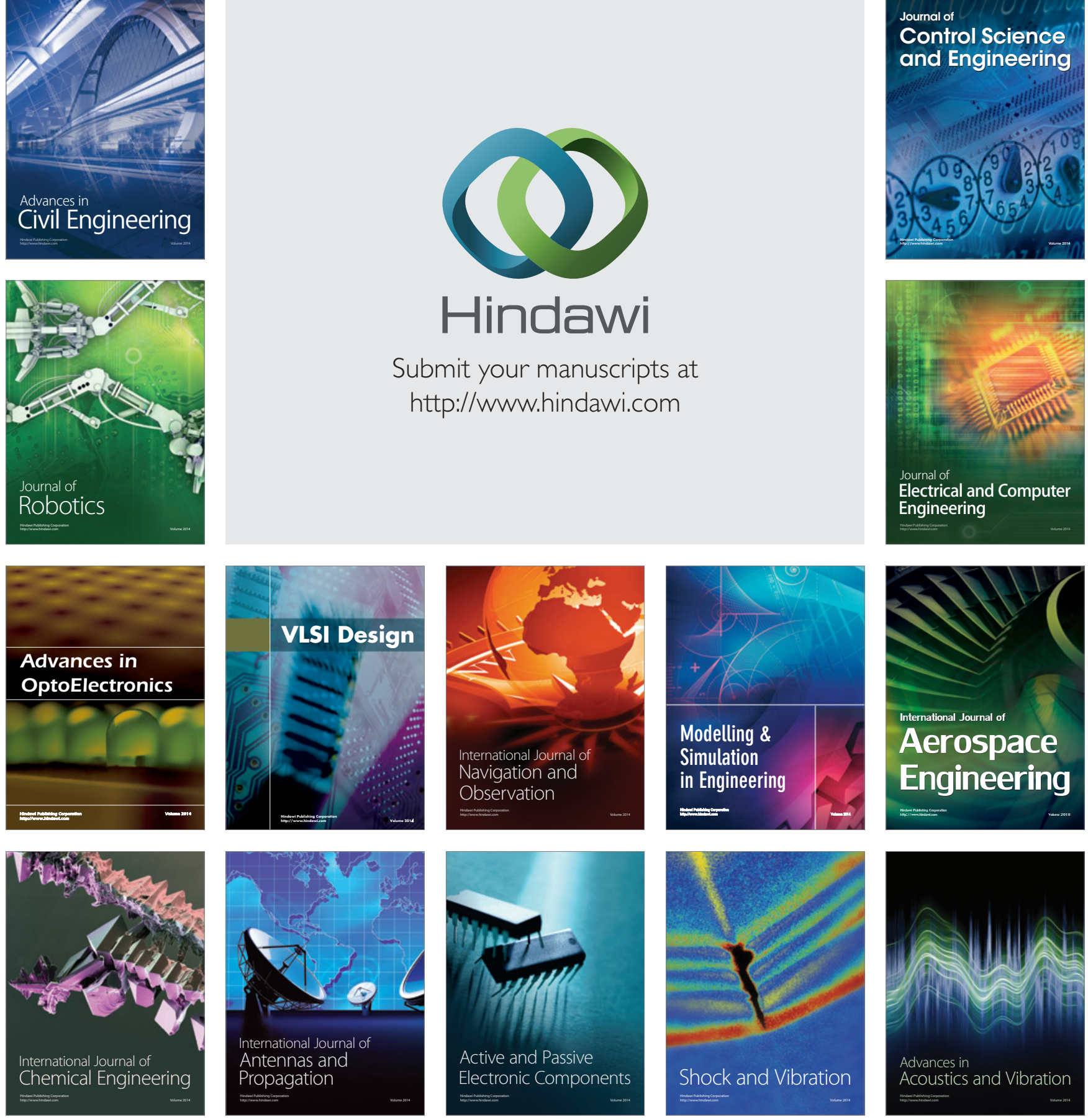